\title{
Electrical cross-talk in rotating ring-disk experiments
}

\author{
S. Vesztergom ${ }^{\text {a }}$, N. Barankai ${ }^{\mathrm{b}}$, N. Kovács ${ }^{\mathrm{a}}$, M. Ujvári ${ }^{\mathrm{a}}$, P. Broekmann ${ }^{\mathrm{c}}$, \\ H. Siegenthaler ${ }^{\mathrm{c}}$, G.G. Láng*a \\ ${ }^{a}$ Eötvös Loránd University, Institute of Chemistry, Department of Physical Chemistry, \\ ${ }^{b}$ MTA-ELTE Research Group in Theoretical Physics, \\ Pázmány P. sétány 1/A, H-1117 Budapest, Hungary \\ ${ }^{c}$ University of Bern, Department of Chemistry and Biochemistry, \\ Freiestrasse 3, 3012 Bern, Switzerland
}

\begin{abstract}
The electrical cross-talk between the two working electrodes of generator/collector systems is investigated. Digital simulations are carried out to model simple collection experiments with a rotating ring-disk electrode (RRDE) immersed into a finite resistance solution of a redox couple, where electrical cross-talk may arise due to the shared current routes of the two working electrodes. Based on the analysis of the Kirchhoff (Laplace) matrix of the simulation mesh it is demonstrated that the cross-talk effect is heavily influenced by the selection of the reference point for potential measurements; in practice this is the position of the reference electrode or the tip of the Luggin probe. The devised model is validated by means of a simple and demonstrative experiment.
\end{abstract}

Keywords: RRDE, ohmic potential drop, digital simulations, Kirchhoff (Laplace) matrix

\section{Introduction}

In generator/collector (GC) [1] experiments one usually measures stationary detector and collector currents and quantifies the amount of formed products by determining the current ratios. Nowadays, however, transient techniques are also often applied in GC setups like scanning electrochemical microscopy (SECM) [2,3] or the rotating ring-disk electrode (RRDE) [4-7] in order to increase sensitivity or to facilitate the detection of certain products which would otherwise be undetectable by stationary methods. For the same purpose ac-mode

${ }^{*}$ Corresponding author. E-mail: langgyg@chem.elte.hu 
measurements are sometimes also applied in both configurations [8-13] and yield useful information, for example, on surface-adsorbed intermediates.

GC systems in general provide useful means to detect and quantify the products and sideproducts of electrode reactions, yet these methods are not without any pitfalls. The aim of this paper is to point out one of these: a certain interference factor, namely the $I R$-drop related electrical cross-talk that can arise between the current-potential characteristics of the working electrodes in practically any four-electrode configurations [14-19]. Cross-talk originates from the shared current routes of the two working electrodes and causes an uncompensated potential shift at one electrode, depending on the current flow of the other one. Cross-talk can, in many cases, lead to serious misinterpretations of the current signals measured in 4electrode systems. This is especially true if transient perturbations resulting in high currents are applied to either one or both of the working electrodes.

In this paper we propose a digital simulation model of the RRDE system which can account for IR-drop related cross-talk and its influence on the results of electrochemical collection experiments. This model can handle any arbitrary potentiostatic (or potentiodynamic) perturbations, and it can also be used to describe four-electrode systems other than the RRDE. After a brief introduction to the theory we present the results of test simulations which are validated by means of a demonstrative experiment. Based on simulation results we define diagnostic criteria for the detection of electrical cross-talk. Strategies for the elimination of cross-talk and/or the correction of measured data are also suggested.

\section{Theory}

Several simulation methods were described in the past to study the RRDE system numerically [20-23]. In [23] it was showed that by applying a smooth spatial discretization (a tiling of the space under the RRDE tip into small annulus-shaped cells), and by an accurate numerical solution of the Navier-Stokes equations describing hydrodynamic flow [23,24] it is possible to simulate simple RRDE experiments that very well satisfy some basic theoretical predictions. I.a., it was shown [23] that for a simple collection experiment the simulated limiting currents for the disk closely match the predictions of the Levich equation [25], and there is also a good agreement between simulated and theoretical [26] collection efficiencies.

In the following we show that by solving the equations of Kirchhoff's circuit laws over a discrete mesh representing the RRDE system [23] it is possible to carry out simulations that 
account for the effects of cross-talk on electrochemical collection experiments. We confine our attention to a system containing two electro-active species (Red and Ox) which, at the working electrodes, can undergo the reaction

$$
\mathrm{Ox}+\mathrm{e}^{-} \rightleftharpoons \mathrm{Red} .
$$

We assume that this reaction proceeds reversibly on both the disk and the ring, and thus the concentrations of the Red and Ox species at the vicinities of the working electrodes are always in accordance with the Nernst equation:

$$
\frac{c_{\text {Ox neardiskor ring }}}{c_{\text {Red near diskor ring }}}=\exp \left[\frac{\left(\widetilde{E}_{\text {diskor ring }}-E^{\Theta}\right) F}{R T}\right] \text {, }
$$

where $\widetilde{E}_{\text {diskorring }}$ is a corrected value of the applied potential $E_{\text {diskorring }}$ :

$$
\widetilde{E}_{\text {diskor ring }}=E_{\text {diskor ring }}-\delta_{I R, \text { diskor ring }} \text {, }
$$

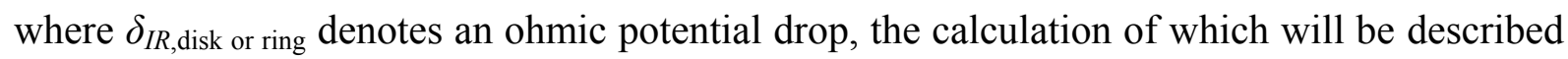
later on. Knowing the concentration changes in the finite volume simulation cells and the simulation time step $\Delta t$ the currents flowing through the working electrodes ( $I_{\text {disk }}$ and $\left.I_{\text {ring }}\right)$ can be determined:

$$
I_{\text {diskor ring }}=F \Delta t \sum_{\substack{\text { simulationcells } \\ \text { neardiskor ring }}}\left(c_{\text {ox cell }}(t)-c_{\text {Ox in cell }}(t-\Delta t)\right) V_{\text {cell }} .
$$

As in [23] we apply the method of finite volumes in order to simulate the RRDE system. Both spatial and temporal discretizations are applied, and the algorithm proceeds iteratively. In each time step $\Delta t$ the boundary conditions according to equation (2) are realized, which changes the concentrations in the electrode-neighbouring cells of the simulation mesh. The concentration changes are then propagated throughout the entire system as governed by the (discretized) equations of mass transport. (Details about solving the discretized transport equations are given in [19].) The simulation of cross-talk effects is added to this scheme by implementing a downhill simplex root-finding algorithm [27] using which in each simulation step appropriate values of $\widetilde{E}_{\text {disk }}$ and $\widetilde{E}_{\text {ring }}$ are determined. These, when used in Equations (2) and (4), give rise to $I_{\text {disk }}$ and $I_{\text {ring }}$ currents that in turn establish ohmic potential drops $\left(\delta_{I R \text {, disk }}\right.$ and $\delta_{I R \text {,ring }}$ ) satisfying Equation (3). Based on $I_{\text {disk }}$ and $I_{\text {ring }}, \delta_{I R \text {, disk }}$ and $\delta_{I R \text {, ring }}$ are determined by solving Kirchhoff's current laws over the simulation mesh, as it will be shown below. 
As a first step, we interpret our simulation mesh as a network of electrical resistors; this concept is illustrated by the graphical abstract of this paper. In case we have a total number of $m$ simulation cells in the mesh, the -equialent circuit" will contain $n=m+3$ equipotential nodes due to the three metallic conductors (the disk, the ring and the auxiliary electrode) present in the system. The electric potentials of each node will be ordered in the vector $\boldsymbol{\psi}$; the currents leaving or entering the circuit at each node will be ordered in the vector $\boldsymbol{t}$. Since current cannot enter or leave the system, except through the aforementioned electrode metals, $\boldsymbol{l}$ will have only three non-zero entries, the sum of which is 0 .

There are two types of conductances which can be identified in an equivalent circuit of a simulation mesh. Between each neighbouring cells of the solution, bulk conductance values are defined by the $\kappa$ bulk conductivity of the solution and the geometric parameters of the simulation cells as

$$
\sigma=\kappa \frac{A}{d}
$$

where $A$ is the area of the surface between the cells and $d$ the distance of their centre points. We assume that the bulk conductivity $\kappa$ is constant for the entire system and has no spatial or temporal dependence; i.e., we only consider strongly supported electrolyte solutions.

Aside from bulk conductances there are also interfacial conductances present in the equivalent circuit. Although in principle these could be finite (and could even change as the simulation proceeds), here we will assume that their values are infinitely high. This is in agreement with the assumption that all electrode reactions are reversible in the system.

Based on the above considerations, all conductances present in the system are known, and we can construct the $n \times n$ Kirchhoff matrix ${ }^{1} \mathrm{~K}$ defined as

$$
\mathrm{K}_{(j, k)}=\left\{\begin{array}{cc}
\sigma_{(j, k)} & \text { if } j \neq k \\
-\sum_{j \neq i} \sigma_{(j, i)} & \text { if } j=k
\end{array}\right.
$$

In Equation (6) the term $\sigma_{(j, k)}$ denotes a conductance which directly links nodes $j$ and $k$ of the equivalent circuit. In order to estimate the ohmic drop affecting the two working electrodes, the matrix-vector representation of the equations of Kirchhoff's current-laws,

$$
\mathrm{K} \psi=\boldsymbol{\imath},
$$

\footnotetext{
${ }^{1}$ In the context of graph theory, the Kirchhoff matrix is often alternatively called the - weighted Laplace matrix.
} 
has to be solved for $\psi$. As the inverse of $K$ does not exist [24], we calculate the generalized inverse [28] $\mathrm{K}^{\dagger}$ to obtain the vector of electric potentials as

$$
\boldsymbol{\psi}=\mathrm{K}^{\dagger} \boldsymbol{l}
$$

Using the $\mathrm{K}^{\dagger}$ matrix it is then possible to obtain the $\psi$ vector in each iteration step from Equation (8). Note that $\psi$ is only determined up to an additive constant, which is due to the fact that $\mathrm{K}$ is a positive semi-definite matrix that has exactly one zero eigenvalue with a corresponding element-wise constant eigenvector. Thus the $\delta_{I R \text {,disk }}$ and $\delta_{I R \text {,ring }}$ terms introduced in Equation (2) are to be defined in the form of a difference:

$$
\begin{aligned}
& \delta_{I R, \text { disk }}=\psi_{\text {disk }}-\psi_{r}, \\
& \delta_{I R, \text { ring }}=\psi_{\text {ring }}-\psi_{r},
\end{aligned}
$$

where $r\left(1 \leq r \leq \mathrm{n}^{2}\right)$ is the index of the equipotential node that we choose as the reference point for voltage measurement. As suggested by Equations (9.a)-(9.b), the position of the reference point (in practice, the location of the tip of the Luggin probe) can have a very deep impact on the current-voltage characteristics of the two working electrodes in RRDE systems.

\section{Experimental}

In order to demonstrate the effect of $I R$-drop related cross-talk on the results of simple collection experiments, measurements and simulations were both carried out. A PINE AFE7R8PtPt RRDE was used and measurements were taking place in a cell that contained a solution of $\mathrm{K}_{3}\left[\mathrm{Fe}(\mathrm{CN})_{6}\right]$ and $\mathrm{K}_{4}\left[\mathrm{Fe}(\mathrm{CN})_{6}\right]$ in equal, $10 \mathrm{mmol} \cdot \mathrm{dm}^{-3}$ concentrations with $0.5 \mathrm{~mol} \cdot \mathrm{dm}^{-3} \mathrm{Na}_{2} \mathrm{SO}_{4}$ as a supporting electrolyte. All chemicals were of purissimum grade and purchased from Reanal, Hungary; ultra-pure (Milli-Q) water was used for preparing the solution. In order to assure that the current distribution is as homogeneous as possible, some $10 \mathrm{~cm}^{3}$ of $\mathrm{Hg}$ was placed in the cell, and this - rarcury lake" was used as an auxiliary electrode. Saturated calomel electrode (SCE) was used as a reference, and was contacted to the working electrode compartment by the use of a Luggin probe ending in a fine capillary. The Luggin probe itself was filled with the same solution as the working electrode compartment. The entire cell was movable under the RRDE tip, which allowed us to choose different positions of the Luggin probe with respect to the disk and ring. Prior to the measurements, the cell was deaerated using Ar gas.

\section{Results and discussion}


Simulations were carried out by assuming that Red and Ox are, similarly to the measurement, present in equal $\left(10 \mathrm{mmol} \cdot \mathrm{dm}^{-3}\right)$ concentrations. An RRDE geometry identical to that of the PINE AFE7R8PtPt tip (used in the experiments) was considered. The standard potential of the electrode reaction according to Equation (1) was assumed to be $E^{\Theta}=0 \mathrm{~V}$ vs. an -deal" reference electrode.

Figure 1 shows the results of collection experiments and of digital simulation. In both cases cyclic voltammograms (sweep rate: $50 \mathrm{mVs}^{-1}$ ) were taken at the disk electrode in a $\pm 325 \mathrm{mV}$ wide potential window around the open circuit potential value. The ring potential was held at $E^{\Theta}$ and the ring currents measured simultaneously with the disk voltammograms were plotted vs. $E_{\text {disk }}$. The rotation rate of the RRDE tip was set to $100 \mathrm{~min}^{-1}$.

Simulations and experiments were both carried out by selecting three different reference points for the voltage measurements. In case of the experiments the Luggin probe was set to different positions as shown by the photographs of Figure 1. In the simulations, different values for the $r$ index in Equations (9.a)-(9.b) were chosen so that the situation would mimic the experimental case, and the reference point for voltage measurement would fall directly under the disk (case 1), directly under the ring (case 2) and under the disk but at some greater distance (case 3). Simulations were also carried out in a cross-talk free case 4.

From Figure 1 it can be seen that the position of the Luggin-probe can have a serious effect on the current responses of the two electrodes. If the Luggin probe is set very close to the disk where high currents flow, the recorded ring signal contains a strong distorting component. Less intense cross-talk can be experienced when the Luggin probe is placed close to the ring, while at high distances almost no cross-talk occurs (although the responses of both electrodes are slightly distorted by a -nanal IR-drop" [18]). The model presented in Section 2 describes well both the cross-talk and the -nomal IR-drop" effects; the results of simulations are in agreement with those of experiment. Based on the results of digital simulations, in what follows we give some rules of thumb that can help experimentalists working with RRDE (or other GC) systems in dealing with the effects of cross-talk:

Diagnostic criteria. A visual comparison of curves recorded with different Luggin probe positions is always advised. Current responses that depend on the position of the Luggin-probe imply the presence of cross-talk. When using an RRDE it may also be expedient to record curves in the absence of rotation: if the polarization of the disk causes high currents to appear also on a stagnant RRDE ring, this is usually an indication of cross-talk (Figure 2(a)). 
Correction of curves measured in the presence of cross-talk. Consider an experiment (or simulation) that is carried out by placing the Luggin probe close to the ring (case 2 in Figure 1). The measured ring response is in this case almost ideal, while the disk voltammogram is slightly distorted by cross-talk. However, if one determines the ohmic resistances $R_{\text {disk toring }}, R_{\text {disk toaux }}$ and $R_{\text {ring to aux }}(e . g$., by means of 2-pole impedance measurements), one can estimate $\delta_{I R \text {, disk }}$ as a function of $I_{\text {disk }}$ and $I_{\text {ring }}$ (Figure 2(b)) and make corrections to the potential scale of the disk voltammograms. Corrected curves are shown in Figure 2(c).

Experimental elimination. Cross-talk (and other $I R$-drop) effects may be experimentally eliminated by using two reference electrodes that target, through two different Luggin probes, the disk and the ring. A suitable bi-potentiostat allowing the connection of two references is needed for this technique. Special care must be taken, however, that hydrodynamic conditions are not disturbed by the probes and no significant screening of the electrodes occur.

\section{Conclusion}

By solving Kirchhoff's equations over a finite volume simulation mesh of the RRDE system the effect of electrical cross-talk on simple collection experiments was studied. The results of digital simulations were validated by a comparison to measured data. Based on the results of digital simulations, diagnostic criteria as well as correction and elimination strategies were suggested. Similar considerations can also hold for other four-electrode configurations such as SECM (and in some cases also electrochemical STM or conductingprobe AFM).

\section{Acknowledgement}

Financial support from the Scientific Exchange Programme NMS ${ }^{\text {ch }}$ (SciEx-13.060), the Hungarian Scientific Research Fund (OTKA-K109036) and the Swiss National Science Foundation (SNSF) is gratefully acknowledged. 


\section{Figure captions:}

Figure 1. Simulated (a) and measured (b) disk voltammograms and ring currents recorded in parallel with them. The disk is polarized in a $\pm 325 \mathrm{mV}$ wide potential window around the open circuit potential at a rate of $50 \mathrm{mVs}^{-1}$. In the meantime the ring is held at $E^{\Theta}$. In cases 1 , 2 and 3 the Luggin probe takes different positions under the RRDE tip, as illustrated by the photos. In case 4 of the simulation no IR-drop effects were taken into account.

Figure 2. (a) In a cross-talk free case the ring signal on an unrotated RRDE should be practically zero: high currents indicate cross-talk. (b) Simplified equivalent circuit of the RRDE system showing the calculation of $\delta_{I R \text {, disk }}[15]$. (c) By shifting the potential scale of the disk CVs with $\delta_{I R, \text { disk }}$, the disk signal may be corrected for cross-talk effects. Simulation results were obtained at a rotation rate of $500 \mathrm{~min}^{-1}$ and a disk sweep rate of $10 \mathrm{mV} \mathrm{s}^{-1}$; other settings were the same as in case 2 of Figure 1(a).

\section{References}

[1] E. O. Barnes, G. E. M. Lewis, S. E. C. Dale, F. Marken, R. G. Compton, Generatorcollector double electrode systems: A review, Analyst 137 (2012) 1068-1081.

[2] Q. Wang, J. Rodríguez-López, A. J. Bard, Reaction of $\mathrm{Br}_{2}$ with adsorbed $\mathrm{CO}$ on Pt, studied by the surface interrogation mode of scanning electrochemical microscopy, $\mathrm{J}$ Am Chem Soc 131 (2009) 17046-17047.

[3] N. Ktari, C. Combellas, F. Kanoufi, Local oxidation of polystyrene by scanning electrochemical microscopy, J Phys Chem C 115 (2011) 17891-17897.

[4] S. Vesztergom, M. Ujvári, G. G. Láng, RRDE experiments with potential scans at the ring and disk electrodes, Electrochem Commun 13 (2011) 378-381.

[5] S. Vesztergom, M. Ujvári, G. G. Láng, RRDE experiments with independent potential scans at the ring and disk electrodes - 3D map of intermediates and products of electrode processes, Electrochem Commun 19 (2012) 1-4.

[6] S. Vesztergom, G. G. Láng, The construction of a novel electrochemical measuring system for enhanced rotating ring-disk electrode experiments, Instrum Sci Technol 41 (2013) 82-95.

[7] S. Vesztergom, M. Ujvári, G.G. Láng, Dual dynamic voltammetry with rotating ringdisk electrodes, in: Y. Saito, T. Kikuchi (Eds.), Voltammetry: Theory, Types and Applications, Nova Science Publishers, New York, 2013.

[8] W. J. Albery, R. G. Compton, A. R. Hillman, Ring-disc electrodes. Part 18 . Collection efficiency for high frequency A.C., Trans Faraday Soc 74 (1978) 1007-1019.

[9] W. J. Albery, A. R. Hillman, Ring-disc electrodes. Part 19. - Adsorption studies at low-frequency A.C., Trans Faraday Soc 75 (1979) 1623-1634. 
[10] N. Benzekri, M. Keddam, H. Takenouti, AC response of a rotating ring-disk electrode: Application to 2-D and 3-D film formation in anodic processes, Electrochim Acta 34 (1989) 1159-1166.

[11] N. Kovács, M. Ujvári, G. G. Láng, P. Broekmann, S. Vesztergom, Characterization of the capacitance of a rotating ring-disk electrode, Instrum Sci Technol 43 (2015) 633648.

[12] D. Trinh, M. Keddam, X. R. Novoa, V. Vivier, Alternating-current measurements in scanning electrochemical microscopy, Part 1: Principle and theory, ChemPhysChem 12 (2011) 2169-2176.

[13] D. Trinh, M. Keddam, X. R. Novoa, V. Vivier, Alternating current measurements in scanning electrochemical microscopy, Part 2: Detection of adsorbates, ChemPhysChem 12 (2011) 2177-2183.

[14] C. Gabrielli, M. Keddam, H. Takenouti, Étude de la répartition du potentiel a la surface d'une électrode a disque-anneau, J Chim Phys Phys Chim Biol 69 (1972) 737-740

[15] M. Shabrang, S. Bruckenstein, Equivalent circuit for the uncompensated resistances occurring at ring-disk electrodes, J Electrochem Soc 121 (1974) 1439-1444.

[16] S. Vesztergom, M. Ujvári, G. G. Láng, Dual cyclic voltammetry with rotating ring-disk electrodes, Electrochim Acta 110 (2013) 49-55.

[17] D. Trinh, E. Maisonhaute, V. Vivier, Electrical cross-talk in transient mode of scanning electrochemical microscopy, Electrochem Commun 16 (2012) 49-52.

[18] C. Dörfel, D. Rahner, W. Forker, Studies of coupling effects and ohmic potential drops at ring-disc electrodes, J Electroanal Chem 107 (1980) 257-270.

[19] A. R. Despić, I. Epelboin, H. Gerischer, M. Keddam, S. Bruckenstein, W. J. Albery, A. J. Bard, J. K. Dohrmann, E. J. Casey, C. L. Gardner, B. Kastening, M. Fleischmann, R. E. W. Jansson, R. D. Rieke, General discussion, Faraday Disc Chem Soc 56 (1973) $367-378$.

[20] K. B. Prater, A. J. Bard, Rotating ring-disk electrodes I. Fundamentals of the digital simulation approach, J Electrochem Soc 117 (1970) 207-213.

[21] S. W. Feldberg, M. L. Bowers, F. Anson, Hopscotch finite difference simulation of the rotating ring-disc electrode, J Electroanal Chem 215 (1986) 11-28.

[22] J. Farkas, L. Kiss, Á. Fóthi, Digital simulation model of the rotating ring-disc electrode. Part 1, Acta Chim Acad Sci Hung 104 (1980) 405-414.

[23] S. Vesztergom, N. Barankai, N. Kovács, M. Ujvári, T. Wandlowski, G. G. Láng, Rotating ring-disk electrode with dual dynamic potential control: Theory and practice, Acta Chim Slov 61 (2014) 223-232.

[24] T. Kármán, Über laminare und turbulente Reibung, Z Angew Math Mech 1 (1921) 233252.

[25] V. G. Levich, Physicochemical Hydrodynamics, Prentice Hall, Englewood Cliffs, NJ, 1962.

[26] A. J. Bard, L. R. Faulkner, Electrochemical methods: Fundamentals and applications, 2nd Edition, Wiley, New York, 2001. 
[27] W. H. Press, S. A. Teukolsky, W. T. Vetterling, B. P. Flannery, In The Art of Scientific Computing, Cambridge University Press, New York, 2007.

[28] A. Ben-Israel, T. N. E. Greville, Generalized Inverses, Springer, Heidelberg, 2003. 
$\left(E_{\text {disk }}\right.$ vs. ref. $) / \mathrm{mV}$

$\left(E_{\text {disk }}\right.$ vs. SCE $) / \mathrm{mV}$
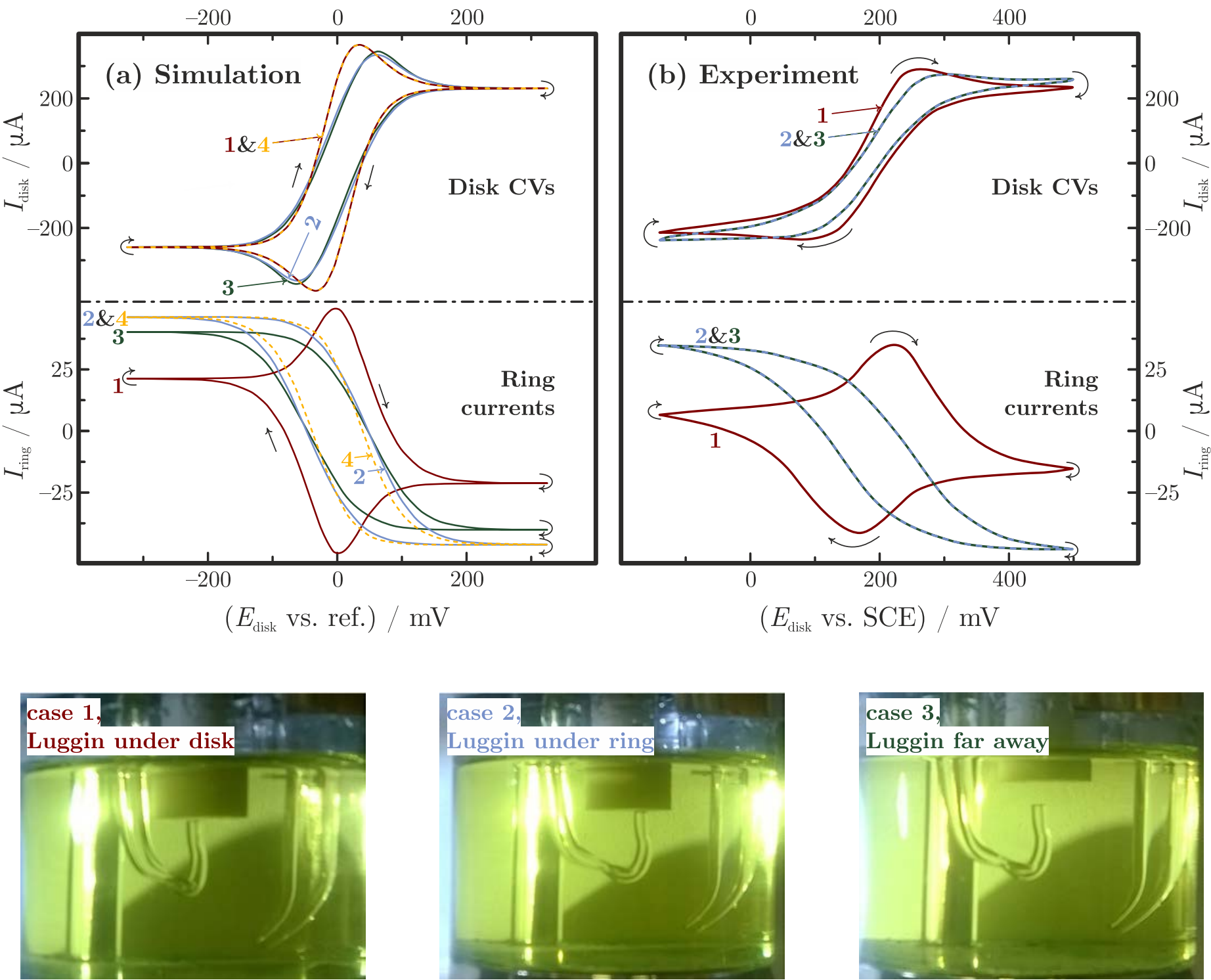
(a)

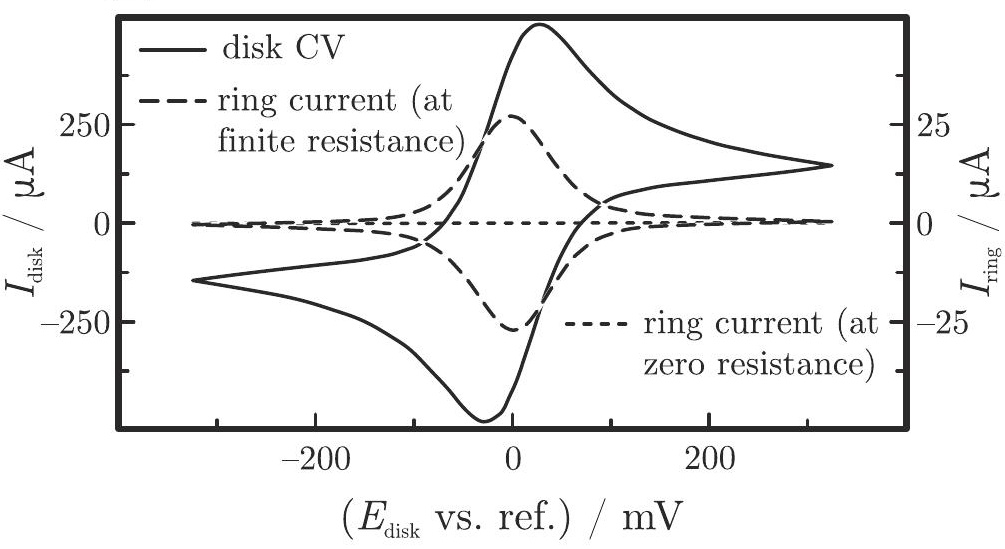

(b)

$$
\begin{aligned}
& R_{1}=\frac{1}{2}\left(R_{\text {disk to aux }}+R_{\text {disk to ring }}-R_{\text {ring to aux }}\right) \\
& R_{2}=\frac{1}{2}\left(-R_{\text {disk to aux }}+R_{\text {disk to ring }}+R_{\text {ring to aux }}\right) \\
& R_{3}=\frac{1}{2}\left(R_{\text {disk to aux }}-R_{\text {disk to ring }}+R_{\text {ring to aux }}\right)
\end{aligned}
$$

Disk

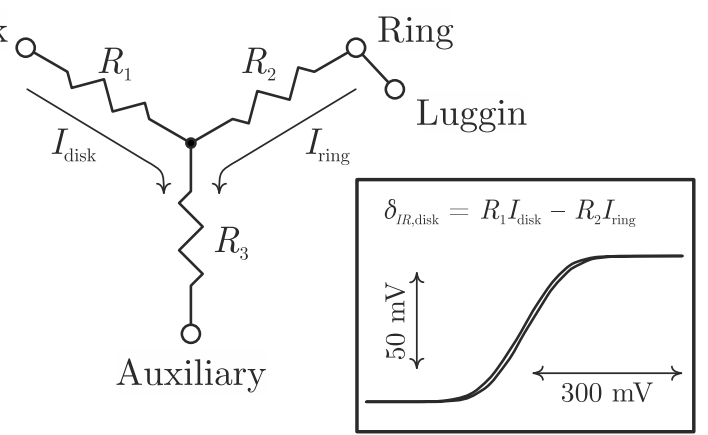

(c)

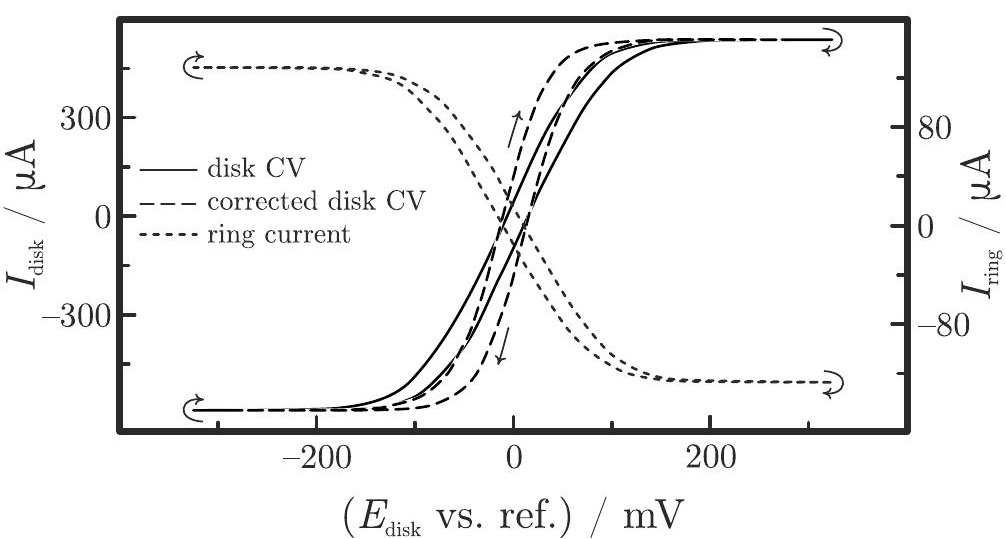


$I_{\text {ring }}=-20 \mu \mathrm{A} \quad I_{\text {disk }}=18 \mu \mathrm{A}$
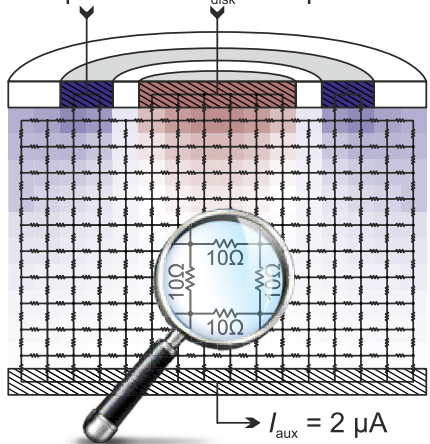

$\gtreqless$

음

草

는

$\frac{3}{2}$

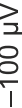

